\title{
Action Spectroscopy of the Isolated Red Kaede Fluorescent Protein Chromophore
}

Neville J. A. Coughlan, ${ }^{1}$ Mark H. Stockett, ${ }^{2}$ Christina Kjær, ${ }^{3}$ Eleanor K. Ashworth, ${ }^{4}$ Philip C. Bulman Page, ${ }^{4}$ Stephen R. Meech, ${ }^{4}$ Steen Brøndsted Nielsen, ${ }^{3}$ Lluís Blancafort, ${ }^{5}$ W. Scott Hopkins, ${ }^{1, \text { a) }}$ and James N. Bull ${ }^{4}$, b)

1) Department of Chemistry, University of Waterloo, Waterloo, Canada

2) Department of Physics, Stockholm University, SE-10691 Stockholm, Sweden

3) Department of Physics and Astronomy, Aarhus University, Aarhus 8000, Denmark

4) School of Chemistry, Norwich Research Park, University of East Anglia, Norwich NR4 7TJ, United Kingdom

${ }^{5)}$ Institut de Química Computacional i Catálisi and Departament de Química, Universitat de Girona, C/M.A.Capmany 69, 17003 Girona, Spain

The incorporation of fluorescent proteins into biochemical systems has revolutionized the field of bioimaging. In a bottom-up approach, understanding the photophysics of fluorescent proteins requires detailed investigations on the light-absorbing chromophore, which can be achieved by studying the chromophore in isolation. This paper reports a photodissociation action spectroscopy study on the deprotonated anion of the red Kaede fluorescent protein chomophore, demonstrating that at least three isomers - assigned to deprotomers - are generated in the gas phase. Deprotomer-selected action spectra are recorded over the $\mathrm{S}_{1} \leftarrow \mathrm{S}_{0}$ band using an instrument with differential mobility spectrometry coupled with photodissociation spectroscopy. The spectrum for the principal phenoxide deprotomer spans the $480-660 \mathrm{~nm}$ range with maximum response at $\approx 610 \mathrm{~nm}$. The imidazolate deprotomer has a blue-shifted action spectrum with maximum response at $\approx 545 \mathrm{~nm}$. The action spectra are consistent with STEOM-DLPNO-CCSD calculations of excitation wavelengths for the deprotomers. A third gas-phase species with a distinct action spectrum is tentatively assigned to an imidazole tautomer of the principal phenoxide deprotomer. The study highlights the need for isomer-selective methods when studying the photophysics of biochromophores possessing several deprotonation sites.

\section{INTRODUCTION}

The discovery of fluorescent proteins and their adaptation to bioimaging has revolutionized the visualization of biological processes, ${ }^{1-6}$ prompting efforts to understand and control the photophysics of photoactive proteins and their light-absorbing chromophore. ${ }^{7-10}$ Green fluorescent protein (GFP) and derivatives are amongst the most widely used fluorescent probes for in vivo imaging due to a high fluorescence quantum yield in a desirable optical window and ease of incorporation into biochemical systems. ${ }^{11}$ The photophysics of GFP are driven by a deprotonated chromophore derived from $p$ hydroxybenzylidene-2,3-dimethylimidazolione ( $p$-HBDI) shown in FIG. 1a. ${ }^{12,13}$

Kaede Fluorescent Protein (KFP) is a green-to-red photoconvertible protein found in the stony coral Trachyphyllia geoffroyi ${ }^{14}$ containing a $p$-HBDI-derived chromophore which fluoresces in the green following the absorption of blue light. Irradiation of KFP with near-UV light $(350-400 \mathrm{~nm})$ induces an irreversible photoconversion to a red fluorescent form (rKFP), ${ }^{15}$ generating the chromophore shown in FIG. 1b. Significantly, absorption of visible light by either KFP or rKFP leads to fluorescence but not photoconversion, allowing KFP to be adapted as an optical highlighter for in vivo imaging. ${ }^{16}$ The desirable fluorescence and photoconversion proper-

\footnotetext{
a)Electronic mail: shopkins@uwaterloo.ca

b)Electronic mail: james.bull@uea.ac.uk
}

ties of Kaede have led to the development of a range of synthetic photoactive proteins, including IrisFP which combines fluorescence properties from the Kaede family and photoisomerization properties from the Dronpa family, allowing for reversible on/off photoswitching through $E-Z$ isomerization. ${ }^{17}$<smiles>CC1=N/C(=C\c2ccc(O)cc2)C(=O)N1C</smiles><smiles>CN1C(=O)/C(=C/c2ccc(O)cc2)N=C1/C=C/c1cnc[nH]1</smiles>

FIG. 1. Model fluorescent protein chromophores: (a) $p$-hydroxybenzylidene-2,3-dimethylimidazolione ( $p$-HBDI), where deprotonation on the hydroxyl group $\left(p-\mathrm{HBDI}^{-}\right)$ serves as a model for the GFP chromophore, (b) 2[(1E)-2-(5-imidazolyl)ethenyl]-4-( $p$-hydroxybenzylidene)-5imidazolinone serves as a model for the rKFP chromophore. In the red Kaede protein, the chromophore in (b) is deprotonated on the hydroxyl group. 
Rapid advances in bioimaging over the last two decades have prompted a range of experimental and theoretical investigations on the fundamental photophysical properties $p$-HBDI-based chromophores. ${ }^{10}$ In this context, the gas phase provides a unique environment for characterizing the chromophore's inherent photophysics owing to the absence of intermolecular interactions, making comparisons between experiment and theory straightforward. ${ }^{18}$ While $p$-HBDI ${ }^{-}$(i.e., deprotonated) has been the subject of several gas-phase action spectroscopy investigations, including informing on the inherent absorption profile, ${ }^{19-23}$ microsolvation, ${ }^{24-26}$ photoisomerization propensity, ${ }^{27}$ and ultrafast excited state dynamics, ${ }^{28-30}$ the photophysical properties of the isolated rKFP chromophore are essentially unstudied. The only exception is a combined photoelectron spectroscopy and electronic structure theory study. ${ }^{31}$ The photoelectron spectroscopy component involved recording spectra for three wavelengths in the UV, accessing excited states higher in energy than the $\mathrm{S}_{1}$ state; anion photoelectron spectroscopy is unsuitable for directly probing electronic excited states situated below the electron detachment threshold, which is the case for the $\mathrm{S}_{1}$ state in the deprotonated rKFP chromophore.

The usual strategy for isolating biochromophore anions for fundamental studies is through electrospray ionization from a polar solvent. ${ }^{32}$ However, the deprotonated rKFP chromophore can exist as either the phenoxide or imidazolate deprotomer, corresponding to deprotonation on either the hydroxyl group or the imidazole ring, respectively (see FIG.1b). In this work, the term deprotomer is defined as a deprotonation isomer, i.e. the same $E / Z$ geometric configuration while 'isomer' refers to the broader variation in atomic configurations. Additionally, there are two possible tautomeric structures for the phenoxide deprotomer depending on the configuration of the imidazole ring. Systematic electrospray ionization studies on prototype para-phenoxide molecules possessing two deprotonation sites have shown that the electrosprayed yield of each deprotomer critically depends on solvent $\mathrm{pH}$, electrospray geometry and needle voltage, desolvation conditions and collisional treatment of ions as they are introduced into vacuum. ${ }^{33,34}$ Because many gas-phase action spectroscopy instruments are custom designed or use different commercial electrospray sources, it is difficult to know whether the deprotomer distribution for one experiment is the same as for another. Furthermore, ion mobility spectrometry studies on $p$-HBDI ${ }^{-}$and other 'fluxional' model photoactive protein chromophores ${ }^{35-37}$ have shown that collisional treatment of ions during the electrospray process can lead to deprotomer scrambling. This is because intramolecular proton migration barriers are usually lower than dissociation energies since complete scission of a bond is not required in intramolecular rearrangements. Because deprotomers often have partially overlapping absorption profiles but distinct excited state dynamics, ${ }^{36,38,39}$ robust gas-phase studies should ensure a known and pure form.
Here, we have used two photodissociation instruments to record action spectra of electrosprayed rKFP chromophore anions. One instrument offers isomer selectivity using differential mobility spectrometry (DMS), although heats the ions to $T \approx 450-500 \mathrm{~K}$ during the isomer separation stage before they are trapped and collisionally cooled to some degree. ${ }^{40}$ The second instrument probes ions at $T \approx 298 \mathrm{~K}$, although does not offer isomer/deprotomer selectivity and requires judicious choice of electrospray conditions (including solvent) to achieve a near pure deprotomer yield. Independent measurements recorded from both of these platforms reveal that at least three gas-phase forms of the deprotonated rKFP chromophore are generated using electrospray ionization, and that the principal phenoxide and imidazolate deprotomers have distinct photodissociation action spectra over the $\mathrm{S}_{1} \leftarrow \mathrm{S}_{0}$ band. The biologically-relevant phenoxide deprotomer is the predominant gas-phase species formed in both experiments.

\section{EXPERIMENTAL METHODS}

Isomer-specific photodissociation action spectra were recorded in a modified DMS-MS device capable of irradiating target anions in the third quadrupole region of a triple quadrupole mass spectrometer with laser light. Complete details of the DMS-MS apparatus are given in Refs. 41-43; for a description of the instrument modifications enabling photodissociation action spectroscopy see Ref. 44. The rKFP chromophore was dissolved in acetonitrile with $0.1 \% \mathrm{NH}_{4} \mathrm{OH}$ (to assist deprotonation) to a concentration of $\approx 1 \mu \mathrm{g} \mathrm{mL}^{-1}$. Electrosprayed ions were carried through the planar DMS cell by dry nitrogen gas doped with acetonitrile or propan-2-ol $(1.5 \% \mathrm{v} / \mathrm{v})$. The yield and interconversion of protomers and presumably deprotomers in DMS cells is known to depend on the protic nature of the mobility modifier, i.e. acetonitrile (non-protic) and propan-2-ol (protic), due to formation of micro-solvated clusters. ${ }^{45-47}$ To acquire ionograms of isomer populations, the separation voltage (SV) applied across the DMS cell was fixed at SV $=3600 \mathrm{~V}$ or $4000 \mathrm{~V}$ and compensation voltage (CV) was scanned between $-25 \mathrm{~V}$ and $0 \mathrm{~V}$. For the photodissociation measurements, the CV was fixed to transmit ions from individual ion populations into a triple-quadrupole mass spectrometer (Q1-Q3). Ions were mass selected in Q1 $(\mathrm{m} / z$ 293), transmitted through Q2 and accumulated in Q3 for $\approx 10 \mathrm{~ms}$. Trapped ions were irradiated with light from a tunable optical parametric oscillator (OPO, Horizon II, Continuum, loosely focused, 2-10 $\mathrm{mJ} \mathrm{pulse}^{-1}$ ) pumped by a pulsed Nd:YAG laser $(10 \mathrm{~Hz}$, Surelite, Continuum) for $\approx 500 \mathrm{~ms}$. The parent ions and any photofragment ions were ejected from the ion trap following a massselective axial ejection method ${ }^{48}$ toward a channeltron ion detector. Ion fragmentation efficiencies were normalized with respect to ion count, OPO fluence, and photon number. 
A separate photodissociation action spectrum for the electrosprayed rKFP chromophore anion was recorded using the Sep1 accelerator mass spectrometer at Aarhus University. ${ }^{49,50}$ The rKFP chromophore was dissolved in methanol with a trace amount of $\mathrm{NH}_{4} \mathrm{OH}$. Electrosprayed anions were accumulated in an octupole ion trap that was emptied every $25 \mathrm{~ms} \mathrm{(40} \mathrm{Hz} \mathrm{repetition} \mathrm{rate).} \mathrm{The} \mathrm{ion}$ bunches were accelerated to a kinetic energy of $\approx 50 \mathrm{keV}$ and mass selected using a bending magnet. An OPO laser system (EKSPLA NT342A, $20 \mathrm{~Hz}$, unfocused) excited every second ion bunch midway along a $2.5 \mathrm{~m}$ linear flight region $\left(10^{-6}\right.$ Torr background pressure). Fragment ions were separated using an electrostatic energy analyzer situated after the laser-ion interaction region and were detected with a channeltron. For a given wavelength, the difference in the number of counts between the 'laser-on' and 'laser-off' injections provided the photoinduced signal. Details of the photofragmentation yield with light fluence are given in the Supplementary Material.

Action spectra recorded in both photodissociation experiments were constructed by monitoring the yield of $m / z 278$, corresponding to loss of a methyl group from the deprotonated rKFP chromophore $(\mathrm{m} / z$ 293). Similar to the situation for $p$-HBDI ${ }^{-},{ }^{19,20,22}$ this channel accounted for more than $95 \%$ of the total photofragmentation yield. Note that because photodissociation was, in all cases, dominated by methyl loss, it is not possible to analyse $m / z$ for photofragments to ascertain deprotonation site.

The absorption spectrum for the deprotonated rKFP chromophore in methanol and water (with a drop of $1 \mathrm{M}$ $\mathrm{NaOH}$ ) was recorded using a PerkinElmer Lambda XLS spectrophotometer and quartz cuvette.

\section{THEORETICAL METHODS}

Electronic structure calculations of molecular properties including geometries, relative energies, and vertical excitation energies were performed using the Gaussian 16.B01, ${ }^{51}$ ORCA 4.2.1, ${ }^{52}$ and MRCC (February 2020 release) software packages. ${ }^{53}$ Geometry optimizations and vibrational frequency calculations were performed at the MP2/aug-cc-pVDZ and $\omega$ B97XD/aug-cc-pVDZ levels of theory. ${ }^{54-56}$ Vertical excitation wavelengths were computed at the $\mathrm{df}-\mathrm{CC} 2 / \mathrm{aug}$-ccpVTZ and STEOM-DLPNO-CCSD/aug-cc-pVDZ levels of theory. ${ }^{57,58}$ Franck-Condon-Herzberg-Teller (FCHT) simulation of absorption spectra were performed at $T=0 \mathrm{~K}$ and $T=298 \mathrm{~K}$ (i.e., including hot band contributions), at the $\omega \mathrm{B} 97 \mathrm{X}-\mathrm{D} /$ aug-cc-pVDZ level of theory using the FCHT framework as implemented in Gaussian 16.B01. ${ }^{59}$ Calculations to model solvation involved geometry optimization at the MP2/aug-cc-pVDZ level of theory including the SMD model for bulk solvation and inclusion of five explicit water molecules. ${ }^{60}$ The thermal Gibbs free energy at $T=298 \mathrm{~K}$ was calculated at the CAM-B3LYP /6-311G** level of theory. ${ }^{61}$

\section{RESULTS AND DISCUSSION}

\section{A. Photodissociation action spectroscopy}

A DMS ionogram for deprotonated rKFP chromophore is shown in FIG. 2a, revealing two clear peaks centered at $\mathrm{CV} \approx-9 \mathrm{~V}$ (peak 1) and $\mathrm{CV} \approx-16 \mathrm{~V}$ (peak 2) and a third shoulder peak centered at $\mathrm{CV} \approx-11 \mathrm{~V}$ (peak 3). These peaks correspond to distinct isomer populations eluting from the DMS cell at specific CV shifts. Ion temperatures in the DMS cell are approximated at $T \approx 471 \mathrm{~K}$ (peak 1 ) and $T \approx 474 \mathrm{~K}$ (peak 2 ), based on earlier studies with thermometer ions. ${ }^{40}$ However, even though the ions are heated in the DMS cell, the selected ion populations are thermalized to some degree during their passage and storage in Q3. The temperature of the ions in the photodissociation region is therefore expected to be closer to room temperature.

Photodissociation action spectra recorded at the three CV's identified in FIG. 2a are shown in FIG. 2b. The action spectrum for peak 1 (blue) spans the 660 $470 \mathrm{~nm}$ range with maximum response at $\approx 600-610 \mathrm{~nm}$. This spectrum resembles the photodissociation spectrum recorded on the Sep1 instrument using basic methanol as the electrospray ionization solvent (FIG. 2c), which has a more distinct peak at $\approx 610 \mathrm{~nm}$. The action spectrum for peak 2 (red) appears blue-shifted with maximum response at $\approx 545 \mathrm{~nm}$ and a shoulder feature at $\approx 600 \mathrm{~nm}$.

At $\mathrm{SV}=3600 \mathrm{~V}$, there was insufficient ion population for peak 3 to record a photodissociation action spectrum. However, increase of the DMS separation voltage to $\mathrm{SV}=4000 \mathrm{~V}$ and use of $\mathrm{N}_{2}+1.5 \%$ propan-2-ol $(\mathrm{v} / \mathrm{v})$ as the DMS buffer gas produced an ionogram showing two clear peaks (see Supplementary Material). Estimated ion temperatures at $\mathrm{SV}=4000 \mathrm{~V}$ are $T \approx 500 \mathrm{~K}$. The photodissocation action spectrum for the higher CV ion population was similar to the red curve in FIG. $2 \mathrm{~b}$ (see Supplementary Material), however, the spectrum for the second ion population at lower $\mathrm{CV}$ is distinct and is shown in green in FIG. 2b. The green spectrum has a wavelength of maximum response at $\approx 570 \mathrm{~nm}$. We expect this spectrum to be associated with the peak 3 ion population in FIG. 2a.

\section{B. Deprotomer assignments}

The potential gas-phase structures of the deprotonated rKFP chromophore have been previously considered in detail. ${ }^{31}$ Following that study, there are three deprotonation patterns: two for oxygen deprotonation (phenoxide), which vary in the nitrogen atom that remains protonated and are labelled O1(-) and O2(-) [see FIG.3], and one for nitrogen deprotonation (imidazolate), labelled $\mathrm{N}(-)$ [see FIG. 3]. We assumed fixed $Z$ and $E$ configurations around the $\mathrm{C}_{8}-\mathrm{C}_{9}$ and $\mathrm{C}_{15}-\mathrm{C}_{17}$ bonds, respectively, and for rotamers considered the cisoid $(\mathrm{c})$ and transoid $(\mathrm{t})$ isomers around the $\mathrm{C}_{11}-\mathrm{C}_{15}$ and $\mathrm{C}_{17}-\mathrm{C}_{18}$ bonds. The 

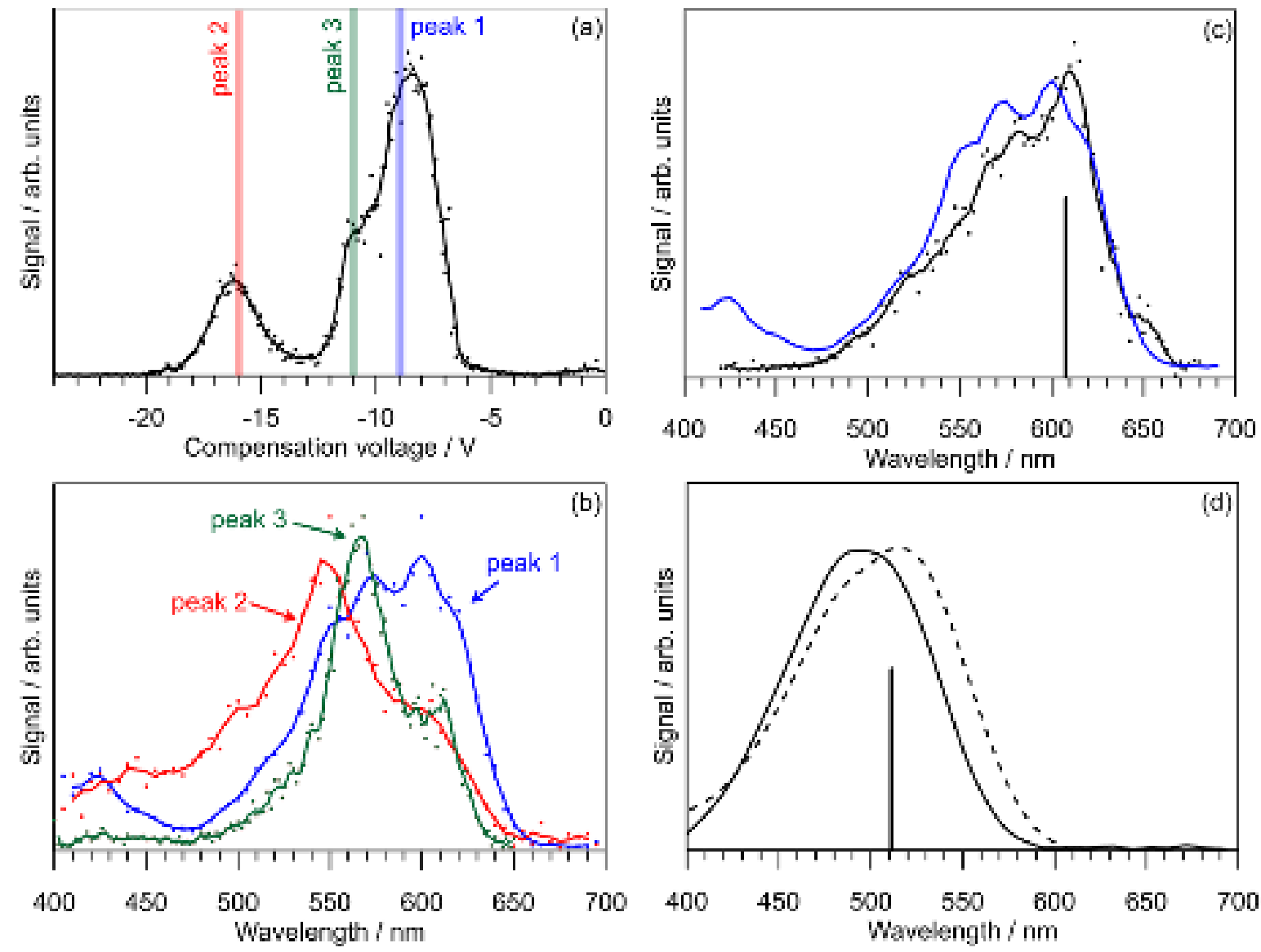

FIG. 2. Action spectroscopy of the deprotonated rKFP chromophore: (a) DMS ionogram recorded using $\mathrm{CH}_{3} \mathrm{CN}_{\text {with }} 0.1 \%$ $\mathrm{NH}_{4} \mathrm{OH}$ as the electrospray solvent and $\mathrm{N}_{2}+1.5 \% \mathrm{CH}_{3} \mathrm{CN}(\mathrm{v} / \mathrm{v})$ as the DMS carrier gas, (b) photodissociation action spectra for ionogram peaks 1 (blue), 2 (red) and 3 (green - see Supplementary Material), (c) photodissociation action spectrum recorded using the Sep1 instrument with basic $\mathrm{CH}_{3} \mathrm{OH}$ electrospray solvent (black) and blue spectrum from (b) for comparison, (d) absorption spectra in basic water (solid) and basic methanol (dashed) solutions. In (b) and (c), light fluence from the OPO laser systems is very low for wavelengths longer than $\approx 680 \mathrm{~nm}$. Vertical bars in (c) and (d) correspond to calculated vertical excitation wavelengths for the $\mathrm{S}_{1} \leftarrow \mathrm{S}_{0}$ transition in the gas phase or in water at the STEOM-DLPNO-CCSD/aug-cc-pVDZ level of theory.

$Z$ configuration for the $\mathrm{C}_{8}-\mathrm{C}_{9}$ bond is known to correspond to the lowest energy geometric isomer for $p$ $\mathrm{HBDI}^{-}{ }^{27}$ Furthermore, the synthesis of the rKFP chromophore was selective for the $Z$ configuration around the $\mathrm{C}_{8}-\mathrm{C}_{9}$ bond - see synthesis details in Ref. 31. Considering the rotamers and imidazole tautomers, this gives a total of 12 isomers. However, many of the rotamers will have low internal rotation barriers, and the corresponding bonds can be considered as free rotors over the timescale of the experiments. ${ }^{36,62}$ The three lowest energy deprotomers are shown in FIG. 3 with calculated relative energies given at the DLPNO-CCSD $(\mathrm{T}) /$ aug-ccpVDZ level of theory. The $\mathrm{N}(-)$ deprotomer is predicted to be the most stable gas-phase speces, with the O1() deprotomer being the most stable phenoxide species, but is situated $2.3 \mathrm{kcal} \mathrm{mol}^{-1}$ above the $\mathrm{N}(-)$ deprotomer (3.6 kcal mol ${ }^{-1}$ at the MP2/aug-cc-pVTZ level of theory in Ref. 31). Because the phenoxide deprotomer is the most stable deprotomer in aqueous solution or methanol (see subsection $\mathrm{C}$ below), we expect that the O1(-) deprotomer is the predominant gas-phase form generated when using 'gentle' electrospray ionization conditions, i.e. weak radio-frequency confinement fields and low ionmolecule impact energies.

To assign the action spectra to specific deprotomers, we calculated $S_{1} \leftarrow S_{0}$ vertical excitation wavelengths for the low energy gas-phase isomers (TABLE I). In all cases, the calculated transition energy for the O1(-) deprotomer is red-shifted (lower transition energy) relative to the N(-) deprotomer. The df-CC2/aug-cc-pVDZ calculations, which predict similar transition wavelengths to earlier MS-CASPT2 $(14,14) / A N O-L$ calculations, ${ }^{31}$ are systemically at shorter wavelengths. Increasing the basis set to aug-cc-pVTZ for the df-CC2 method provided little change to the calculated wavelengths. On the the other hand, the calculated wavelengths at the STEOMDLPNO-CCSD/aug-cc-pVDZ level of theory are in excellent agreement with experiment and support assign- 


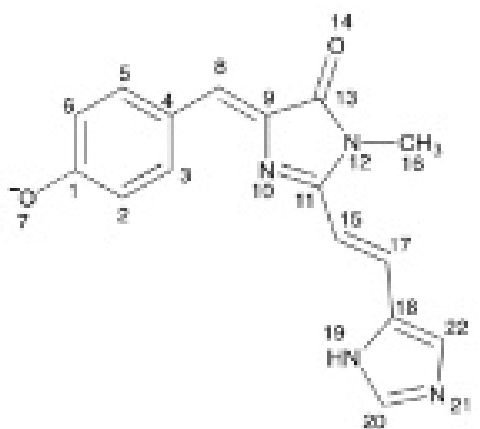

01(-)

$E_{\text {rel }}=2.3 \mathrm{kcal} \mathrm{mol}^{-1}$

(9.8 kJ mol ${ }^{-1}$ )<smiles>COc1ccc(/C=C2\N=C(/C=C/c3c[nH]cn3)N(C)C2=O)cc1</smiles>

O2(-)

$E_{\text {rat }}=5.3 \mathrm{kcal} \mathrm{mol}$.

(22.2 kJ mol')<smiles></smiles>

$\mathrm{N}(-)$

$E_{\text {res }}=0.0 \mathrm{kcal} \mathrm{mol}$ '

FIG. 3. Illustrations of the three lowest energy deprotomers. Relative gas-phase energies $\left(E_{r e l}\right)$ are at the DLPNO$\operatorname{CCSD}(\mathrm{T})$ /aug-cc-pVDZ level of theory with $\mathrm{N}(-)$ being the most stable deprotomer. O1(-) is the most stable phenoxide gas-phase form, possessing s-trans configurations along both $\mathrm{C}_{11}-\mathrm{C}_{15}$ and $\mathrm{C}_{17}-\mathrm{C}_{18}$ bonds. For the action spectra in FIG. $2 \mathrm{~b}$, we assign O1(-) to the blue curve and N(-) to the red curve. We tentatively assign O2(-) to the green curve (peak 3) in FIG. 2b.

ment of ionogram peak 1 in FIG. 2a to the O1(-) deprotomer and peak 2 to the $\mathrm{N}(-)$ deprotomer. The third isomer, corresponding to the green spectrum in FIG. 2b, is tentatively assigned to the $\mathrm{O} 2(-)$ deprotomer, although agreement between the STEOM-DLPNO-CCSD/aug-ccpVDZ calculated wavelength and experiment is not as good as for the other two deprotomers - see Supplementary Material for further discussion. We do not expect $Z$ isomers about the $\mathrm{C}_{15}-\mathrm{C}_{17}$ bond to survive electrospray ionization or the DMS cell since the starting sample was isomerically pure and $Z$-isomers are situated much higher in energy $\left(>10 \mathrm{kcal} \mathrm{mol}^{-1}\right)$.

Franck-Condon-Herzberg-Teller (FCHT) simulations of the absorption spectra for the O1(-) and N(-) species assuming statistical vibrational temperatures of $T=0 \mathrm{~K}$ (black) and $T=298 \mathrm{~K}$ (red) are shown in FIG. 4. The simulated spectra assume the minimum energy geometries; rotamers will broaden the experimental spectra compared with simulation. In both cases, the simulations predict that the $T=298 \mathrm{~K}$ spectra are dominated

\begin{tabular}{|c|c|c|c|c|}
\hline Method & O1(-) & $\mathrm{O} 2(-)$ & $\mathrm{N}(-)$ & $\Delta(\mathrm{eV})$ \\
\hline $\mathrm{df}-\mathrm{CC} 2^{a}$ & $544(547)$ & 558 & $500(503)$ & $0.20(0.20)$ \\
\hline $\mathrm{STEOM}^{b}$ & 608 & 590 & 559 & 0.18 \\
\hline$\omega \mathrm{B} 97 \mathrm{X}-\mathrm{D}$ & 485 & 492 & 453 & 0.18 \\
\hline $\mathrm{MS} \mathrm{CASPT} 2^{c}$ & 554 & - & 499 & 0.25 \\
\hline Experiment & $610 \pm 5$ & - & $545 \pm 5$ & $\approx 0.24$ \\
\hline
\end{tabular}

TABLE I. Calculated vertical excitation wavelengths (in $\mathrm{nm}$ ) for the $\mathrm{S}_{1} \leftarrow \mathrm{S}_{0}$ transition of the O1(-), O2(-) and N(-) deprotomers of the rKFP chromophore. $\Delta$ is the difference between the vertical excitation energies for the O1(-) and $\mathrm{N}(-)$ deprotomers. In all cases the calculated classical oscillator strength, $f>0.8$. ${ }^{a}$ Values in parentheses assume the augcc-pVTZ basis set. ${ }^{b} \mathrm{STEOM}$ is an abbreviation for STEOMDLPNO-CCSD/aug-cc-pVDZ. ${ }^{c}$ MS-CASPT2 $(14,14) /$ ANO-L values from Ref. 31 . by the $0-0$ transition. For O1(-), there is generally good
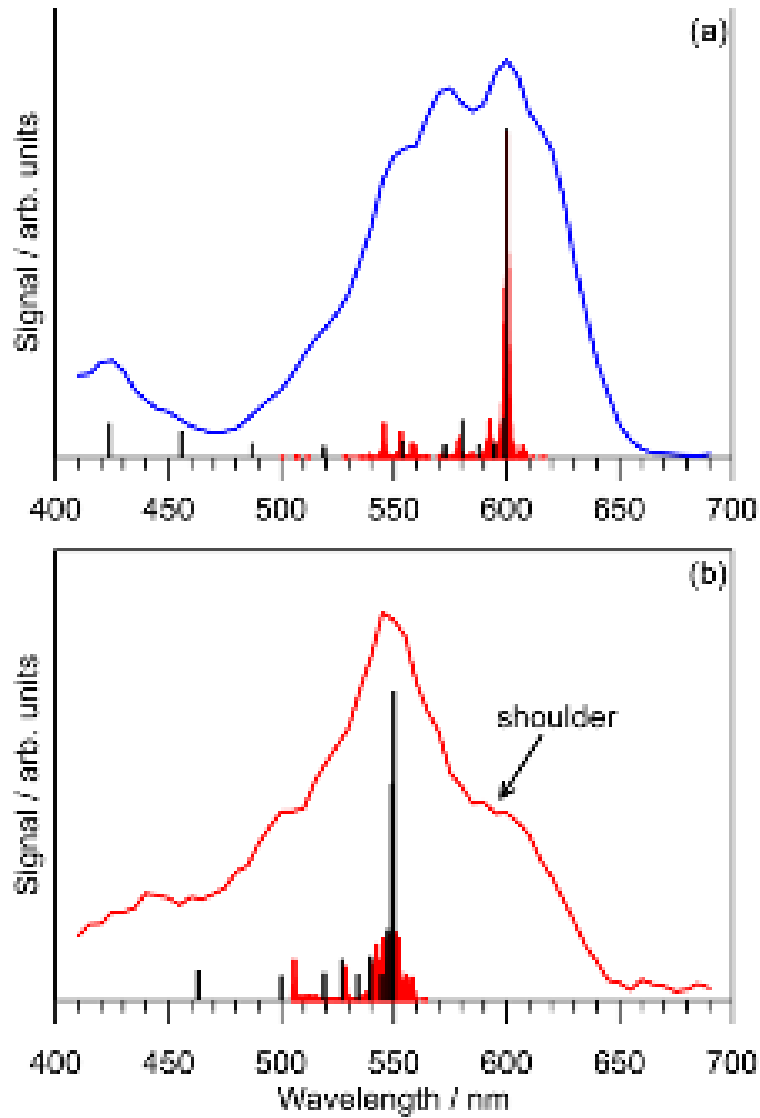

FIG. 4. Franck-Condon-Herzberg-Teller simulations of the $\mathrm{S}_{1} \leftarrow \mathrm{S}_{0}$ absorption profiles for: (a) O1(-) deprotomer, (b) $\mathrm{N}(-)$ deprotomer. In each, the simulated profile has been shifted to agree with experiment (TABLE I). Simulations assume statistical vibrational temperatures of $T=0 \mathrm{~K}$ (black) and $T=298 \mathrm{~K}$ (red) 
agreement between simulation and experiment. For N(), the FCHT simulations do not account for the shoulder at $\approx 600 \mathrm{~nm}$. Before discussing possible explanations, it is worth outlining some details of the photodissociation experiments.

Although photodissociation is the most common gasphase action spectroscopy strategy applied to electrosprayed ions, the dissociation thresholds of many target molecules are situated higher in energy than the electronic transitions of interest, requiring absorption of several photons to produce a photoresponse. As for $p$ $\mathrm{HBDI}^{-},{ }^{19,20,22}$ the principal photodissociation channel for the deprotonated rKFP chromophore involves loss of a methyl group, yielding anions at $m / z 278$. Calculated $\mathrm{N}_{12}-\mathrm{C}_{16}$ adiabatic bond dissociation energies (BDEs) for the $\mathrm{O} 1(-)$ and $\mathrm{N}(-)$ species are $2.40 \mathrm{eV}$ and $3.15 \mathrm{eV}$, respectively, and we expect similar BDEs for any $E$ - $Z$ geometric photoisomers. However, in a photodissociation process requiring sequential absorption of two or more photons, the first photon may induce a photoisomerization with absorption of subsequent photons providing sufficient internal energy to result in dissociation (presumably following internal conversion on the ground state potential energy surface). Using photoisomerization action spectroscopy, we have shown that single-photon induced isomerization, tautomerization and intramolecular proton transfer are common processes in fluxional biochromophores, including for $p$-HBDI- ${ }^{27,35-37,62}$ Another consideration is that the DMS-MS and Sep1 experiments involve different ion irradiation and photofragment detection conditions, which may affect the appearance of the action spectra. In particular, selected ion populations in the DMS-MS instrument are irradiated in a quadrupole ion trap for $\approx 500 \mathrm{~ms}(10 \mathrm{~Hz}$ laser, double pass beam so that ions are exposed to $\approx 10$ light pulses) before being ejected to a detector. In contrast, the Sep1 experiment detects photofragment ions formed several tens of microseconds after interaction with a single light pulse ( $\approx 5 \mathrm{~ns}$ duration). In a multi-photon absorption experiment, ground state statistical rearrangement processes thus have much longer time window to occur in the DMSMS experiment before sufficient photons are absorbed to induced dissociation. This reasoning may account for deviation between the DMS-MS and Sep1 spectra in the 410-480 $\mathrm{nm}$ range in FIG. 2c.

We surmise two possible explanations for the feature at $\approx 600 \mathrm{~nm}$ in the $\mathrm{N}(-)$ action spectrum:

(1) isomerization or deprotomer scrambling induced by absorption of a first photon, followed by absorption of the second photon by a different species. In this case, the action spectrum would be a combination of absorption profiles for the parent deprotomer and a hot intermediate photoisomer (weighted by the photoisomerization quantum yield).

(2) contamination of the $\mathrm{N}(-)$ deprotomer population, e.g. collision-induced deprotomer scrambling of DMSselected ions during their transit into Q3. Similar arguments can be made for long-wavelength shoulder in the action spectrum tentatively assigned to the O2(-) deprotomer.

In the earlier combined photoelectron spectroscopy and electronic structure study on the deprotonated rKFP chromophore ${ }^{31}$ the electrosprayed ion population was assigned to the imidazolate deprotomer through comparing the high electron kinetic edge of the photoelectron spectra with calculated adiabatic electron affinities for the deprotomers. Although the photoelectron experiment may have indeed produced the N(-) deprotomer using methanol-water electrospray ionization solvent, there are several issues related to using electron detachment thresholds to assign deprotomers that should be outlined: (i) while deprotomers can have distinct electron affinities, they could also have significantly varying direct photodetachment cross-sections such that a small amount of one deprotomer can mask the signal from another deprotomer; (ii) the deprotomers could have distinct absorption spectra in the UV (shown in this work for the $\mathrm{S}_{1} \leftarrow \mathrm{S}_{0}$ band in the visible) leading to different extents of prompt autodetachment signal in the photoelectron spectra, where resonant excitation of a minor deprotomer could mask non-resonant signal from the major deprotomer.

\section{Hydration}

To understand hydration of the deprotonated rKFP chromophore, we took the 12 possible isomers and computed structures including five explicit water molecules plus the SMD solvent continuum model. Relative energies for the hydrated species and the relative population in equilibrium at $T=298 \mathrm{~K}$ estimated assuming a Boltzmann distribution are given in TABLE II. The most stable hydrated species is ccO1(-), whose optimized microhydrated cluster is shown in FIG. 5. This species accounts for $42 \%$ of the population. The second most stable species is also an O1(-) deprotomer. Overall, our calculations predict that $75 \%$ of population will correspond to the O1(-) deprotomer, distributed over the $\mathrm{cc}$ and tt rotamers. The rotamers of the O2(-) tautomer account for $21 \%$ of the population, and the $\mathrm{N}(-)$ deprotomer only accounts for a few percent. In the gas phase, the tcN(-) form (FIG. 3) is the most stable species, and the most stable O1(-) form lies more than $2 \mathrm{kcal} \mathrm{mol}^{-1}$ higher in energy. Thus, compared to our present gas-phase calculations and those in Tay et al. ${ }^{31}$, aqueous hydration inverts the preference for the deprotonation site. Preference for the $\mathrm{N}(-)$ deprotomer in solution is obtained when using only the SDM model to treat hydration, i.e., no explicit water molecules. This suggests that the stabilization of the $\mathrm{O} 1(-)$ form in water is primarily due to microhydration effects associated with the hydrogen bonding pattern for the different deprotonation states. Although pure water is uncommon as an electrospray solvent, we expect similar conclusions for the stability of the phenoxide deprotomer in methanol or 50:50 methanol to water (com- 


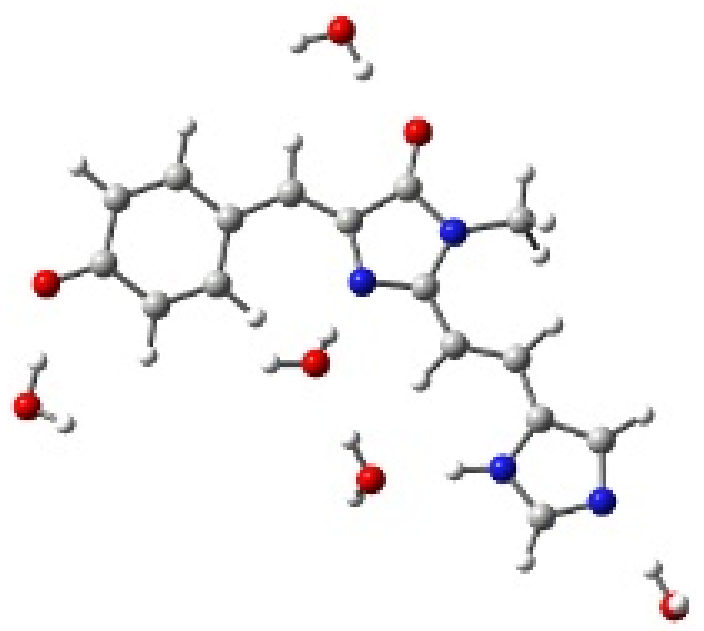

FIG. 5. Optimized structure (SMD-MP2/aug-cc-pVDZ) for the most stable microhydrated [ccO1(-)] deprotomer. The water molecules are hydrogen bonded to the three deprotonation sites $\left(\mathrm{O}_{7}, \mathrm{~N}_{19}\right.$ and $\left.\mathrm{N}_{21}\right)$ as well as to $\mathrm{N}_{10}$ and $\mathrm{O}_{14}$ (see atom numbering in FIG. 3), where the negative charge is localized through resonance.

mon electrospray ionization solvents) due to similarity of the solution absorption spectra (FIG. 2d).

Comparison of the gas-phase action spectrum for the phenoxide deprotomer (FIG. 2c) and the aqueous absorption spectrum (FIG. 2d) reveal a strong hypsochromatic shift $(610 \mathrm{~nm}$ to $490 \mathrm{~nm})$, corresponding to an energy shift of $\approx 0.50 \mathrm{eV}$. This is similar to that for $p$-HBDI ${ }^{-}$ at $\approx 0.41 \mathrm{eV} .{ }^{19}$ The hypsochromatic shift is reproduced in STEOM-DLPNO-CCSD/aug-cc-pVDZ (+SMD) calculations for the ccO1(-) hydrated species shown in FIG. 5, providing the vertical excitation wavelength of $511 \mathrm{~nm}$ (vertical bar in FIG.2d). The calculated value for the

\begin{tabular}{ccc}
\hline Species & $E_{r e l}$ & Population \\
\hline ccO1(-) & 0.0 & $42 \%$ \\
ttO1(-) & 0.1 & $33 \%$ \\
$\operatorname{ccO} 2(-)$ & 0.6 & $14 \%$ \\
ttO2(-) & 1.4 & $4 \%$ \\
tcO2(-) & 1.6 & $3 \%$ \\
$\operatorname{tcO} 1(-)$ & 2.1 & $1 \%$ \\
$\operatorname{ccN}(-)$ & 2.2 & $1 \%$ \\
$\operatorname{ttN}(-)$ & 2.2 & $1 \%$ \\
$\operatorname{tcN}(-)$ & 2.6 & $<1 \%$ \\
$\operatorname{ctO} 2(-)$ & 4.0 & $<1 \%$ \\
$\operatorname{ctO} 1(-)$ & 4.7 & $<1 \%$ \\
$\operatorname{ctN}(-)$ & 5.4 & $<1 \%$ \\
\hline
\end{tabular}

TABLE II. Relative energies, $E_{r e l}$ in $\mathrm{kcal} \mathrm{mol}^{-1}$ of deprotonated rKFP chromophore species in water (SDMMP2/aug-cc-pVDZ) and estimated populations in equilibrium at $T=298 \mathrm{~K}$. $\mathrm{t}$ and $\mathrm{c}$ refer to transoid or cisoid configurations around the $\mathrm{C}_{11}-\mathrm{C}_{15}$ and $\mathrm{C}_{17}-\mathrm{C}_{18}$ bonds (FIG. 3). co-existing ttO1(-) species is $512 \mathrm{~nm}$. The consistency between theory and experiment for gas-phase and aqueous deprotonated rKFP chromophore further supports the above deprotomer assignments of the gas-phase action spectra.

\section{CONCLUSIONS}

This study has demonstrated the capacity for DMS-MS coupled with laser spectroscopy to separate deprotomeric forms the red Kaede fluorescent protein chromophore and record deprotomer-selected photodissociation action spectra. The chromophore predominantly exists as the biologically-relevant phenoxide deprotomer in aqueous solution, and is readily transferred into vacuum assuming 'gentle' electrospray ionization conditions. The photodissociation action spectrum for the $\mathrm{S}_{1} \leftarrow \mathrm{S}_{0}$ transition in the principal phenoxide deprotomer spans the 480 $660 \mathrm{~nm}$ range with maximum response at $\approx 610 \mathrm{~nm}$ and is substantially red-shifted compared with the condensedphase absorption spectrum. The imidazolate deprotomer has an overlapping but blue-shifted photodissociation action spectrum. Significantly, because the action spectrum for the phenoxide deprotomer is red-shifted relative to that for the imidazolate deprotomer, there is a small wavelength window (viz. 600-640 nm) in which femtosecond pump-probe experiments could probe the excited state dynamics of the phenoxide deprotomer in the gas phase. We intend to perform such pump-probe experiments as well as photoisomerization action spectroscopy on the deprotomer-selected forms in order to characterize the isomer-specific detailed excited state dynamics of the $\mathrm{S}_{1}$ state, including lifetimes and $E \rightarrow Z$ isomerization propensities about each double bond.

The preponderance for coexisting gas-phase deprotomers (or protomers in positive mode electrospray ionization) is a common issue for many model biochromophores and will likely become an increasingly important consideration as gas-phase action spectroscopies are applied to more complex biomolecules since they invariably have further sites for deprotonation (or protonation). Robust action spectroscopy studies on such species should have provisions for isomer/deprotomer selectivity.

\section{SUPPLEMENTARY MATERIAL}

The Supplementary Material contains details on: (1) DMS-MS ionogram at $\mathrm{SV}=4000 \mathrm{~V}$ and photodissociation action spectra, (2) photodissociation yield with light fluence for the Sep1 measurements, and (3) optimized geometries for deprotonated rKFP chromophore hydrated complexes. 


\section{DATA AVAILABILITY}

The data that support the findings of this study are available from the corresponding author upon reasonable request.

\section{ACKNOWLEDGMENTS}

Funding was provided by the Swedish Foundation for International Cooperation in Research and Higher Education (STINT) Grant for Internationalisation programme (PT2017-7328 to MHS and JNB), a start-up grant at University of East Anglia (to JNB), a NSERC Discovery Grant and a Collaborative Research and Development grant (to WSH), and provision of computational support from Compute Canada. NJAC acknowledges a Vanier-Banting Postdoctoral Fellowship from NSERC. WHS acknowledges an Early Researcher Award from the province of Ontario. LB thanks the Ministerio de Ciencia, Innovación y Universidades (Spain), project PID-2019-104654GB-I00. SBN acknowledges generous support from the Novo Nordisk Foundation (NNF20OC0064958). SRM and PCBP acknowledge funding from EPSRC through grant EP/H025715/1. Electronic structure calculations were in part carried out on the High Performance Computing Cluster supported by the Research and Specialist Computing Support service at the University of East Anglia. Marlyn Mwita is thanked for recording some preliminary DMS ionograms.

\section{CONFLICTS OF INTEREST}

The authors have no conflicts to disclose.

${ }^{1}$ M. Chalfie, Y. Tu, G. Euskirchen, W. Ward, and D. Prasher, Science 263, 802 (1994).

${ }^{2}$ J. Lippincott-Schwartz, Science 300, 87 (2003)

${ }^{3}$ K. A. Lukyanov, D. M. Chudakov, S. Lukyanov, and V. V. Verkhusha, Nat. Rev. Mol. Cell Biol. 6, 885 (2005).

${ }^{4}$ N. C. Shaner, G. H. Patterson, and M. W. Davidson, J. Cell Sci. 120, 4247 (2007).

${ }^{5}$ D. M. Chudakov, M. V. Matz, S. Lukyanov, and K. A. Lukyanov, Physiol. Rev. 90, 1103 (2010).

${ }^{6}$ G. Cox, ed., Fundamentals of Fluorescence Imaging (Jenny Stanford Publishing, 2019)

${ }^{7}$ A. B. Cubitt, R. Heim, S. R. Adams, A. E. Boyd, L. A. Gross, and R. Y. Tsien, Trends Biochem. Sci. 20, 448 (1995).

${ }^{8}$ J. Zhang, R. E. Campbell, A. Y. Ting, and R. Y. Tsien, Nat. Rev. Mol. Cell Biol. 3, 906 (2002).

${ }^{9}$ M. Zimmer, Chem. Rev. 102, 759 (2002).

${ }^{10}$ S. R. Meech, Chem. Soc. Rev. 38, 2922 (2009)

${ }^{11}$ R. Y. Tsien, Ann. Rev. Biochem. 67, 509 (1998)

${ }^{12}$ R. Heim, D. C. Prasher, and R. Y. Tsien, Proc. Nat. Acad. Sci 91, 12501 (1994).

${ }^{13}$ M. Orm, A. B. Cubitt, K. Kallio, L. A. Gross, R. Y. Tsien, and S. J. Remington, Science 273, 1392 (1996).

${ }^{14}$ R. Ando, H. Hama, M. Yamamoto-Hino, H. Mizuno, and A. Miyawaki, Proc. Nat. Acad. Sci. 99, 12651 (2002).

${ }^{15}$ H. Mizuno, T. K. Mal, K. I. Tong, R. Ando, T. Furuta, M. Ikura, and A. Miyawaki, Mol. Cell 12, 1051 (2003).
${ }^{16}$ M. Tomura, N. Yoshida, J. Tanaka, S. Karasawa, Y. Miwa, A. Miyawaki, and O. Kanagawa, Proc. Nat. Acad. Sci. 105, 10871 (2008).

${ }^{17}$ V. Adam, M. Lelimousin, S. Boehme, G. Desfonds, K. Nienhaus M. J. Field, J. Wiedenmann, S. McSweeney, G. U. Nienhaus, and D. Bourgeois, Proc. Nat. Acad. Sci. 105, 18343 (2008).

${ }^{18} \mathrm{~S}$. Brøndsted Nielsen and J. A. Wyer, eds., Photophysics of Ionic Biochromophores (Springer, 2013).

${ }^{19}$ S. B. Nielsen, A. Lapierre, J. U. Andersen, U. V. Pedersen, S. Tomita, and L. H. Andersen, Phys. Rev. Lett. 87, 228102 (2001).

${ }^{20}$ M. W. Forbes and R. A. Jockusch, J. Am. Chem. Soc. 131, 17038 (2009).

${ }^{21}$ S. H. M. Deng, X.-Y. Kong, G. Zhang, Y. Yang, W.-J. Zheng, Z.-R. Sun, D.-Q. Zhang, and X.-B. Wang, J. Phys. Chem. Lett. 5, $2155(2014)$

${ }^{22}$ A. V. Bochenkova, B. Klaerke, D. B. Rahbek, J. Rajput Y. Toker, and L. H. Andersen, Angew. Chem. Int. Ed. 53, 9797 (2014)

${ }^{23}$ W. Zagorec-Marks, M. M. Foreman, J. R. R. Verlet, and J. M Weber, J. Phys. Chem. Lett. 10, 7817 (2019).

${ }^{24}$ K. Bhaskaran-Nair, M. Valiev, S. H. M. Deng, W. A. Shelton, K. Kowalski, and X.-B. Wang, J. Chem. Phys. 143, 224301 (2015).

${ }^{25}$ J. Langeland, C. Kjær, L. H. Andersen, and S. Brøndsted Nielsen, ChemPhysChem 19, 1686 (2018).

${ }^{26}$ W. Zagorec-Marks, M. M. Foreman, J. R. R. Verlet, and J. M. Weber, J. Phys. Chem. Lett. 11, 1940 (2020).

${ }^{27}$ E. Carrascosa, J. N. Bull, M. S. Scholz, N. J. A. Coughlan, S. Olsen, U. Wille, and E. J. Bieske, J. Phys. Chem. Lett. 9 2647 (2018).

${ }^{28}$ C. R. S. Mooney, D. A. Horke, A. S. Chatterley, A. Simperler, H. H. Fielding, and J. R. R. Verlet, Chem. Sci. 4, 921 (2013).

${ }^{29}$ C. W. West, J. N. Bull, A. S. Hudson, S. L. Cobb, and J. R. R Verlet, J. Phys. Chem. B 119, 3982 (2015).

${ }^{30}$ A. Svendsen, H. V. Kiefer, H. B. Pedersen, A. V. Bochenkova and L. H. Andersen, J. Am. Chem. Soc. 139, 8766 (2017)

${ }^{31}$ J. Tay, M. A. Parkes, K. Addison, Y. Chan, L. Zhang, H. C. Hailes, P. C. Bulman Page, S. R. Meech, L. Blancafort, and H. H. Fielding, J. Phys. Chem. Lett. 8, 765 (2017)

${ }^{32}$ J. Fenn, M. Mann, C. Meng, S. Wong, and C. Whitehouse, Science 246, 64 (1989)

${ }^{33}$ D. Schröder, M. Buděšínský, and J. Roithová, J. Am. Chem. Soc. 134, 15897 (2012).

${ }^{34}$ H. Xia and A. B. Attygalle, Anal. Chem. 88, 6035 (2016).

${ }^{35}$ J. N. Bull, E. Carrascosa, L. Giacomozzi, E. J. Bieske, and M. H. Stockett, Phys. Chem. Chem. Phys. 20, 19672 (2018)

${ }^{36}$ J. N. Bull, G. da Silva, M. S. Scholz, E. Carrascosa, and E. J. Bieske, J. Phys. Chem. A 123, 4419 (2019).

${ }^{37}$ J. N. Bull, J. T. Buntine, E. Carrascosa, M. H. Stockett, and E. J. Bieske, Eur. Phys. J. D 75 (2021).

${ }^{38}$ J. N. Bull, C. S. Anstöter, and J. R. R. Verlet, Nat. Comm. 10 5820 (2019).

${ }^{39}$ J. N. Bull, C. S. Anstöter, and J. R. R. Verlet, J. Phys. Chem. A 124, 2140 (2020).

${ }^{40}$ C. Ieritano, J. Featherstone, A. Haack, M. Guna, J. L. Campbell, and W. S. Hopkins, J. Am. Chem. Mass Spectrom. 31, 582 (2020).

${ }^{41}$ J. L. Campbell, M. Zhu, and W. S. Hopkins, J. Am. Soc. Mass Spectrom. 25, 1583 (2014)

${ }^{42}$ C. Liu, Y. Le Blanc, J. Shields, J. Janiszewki, C. Ieritano, G. Ye G. Hawes, S. Hopkins, and J. L. Campbell, Analyst 140, 6897 (2015).

${ }^{43}$ C. Liu, J. C. Y. Le Blanc, B. B. Schneider, J. Shields, J. J. Federico, H. Zhang, J. G. Stroh, G. W. Kauffman, D. W. Kung, C. Ieritano, E. Shepherdson, M. Verbuyst, L. Melo, M. Hasan, D. Naser, J. S. Janiszewski, W. S. Hopkins, and J. L. Campbell, ACS Cent. Sci. 3, 101 (2017).

${ }^{44}$ N. J. Coughlan, P. J. Carr, S. C. Walker, C. Zhou, M. Guna J. L. Campbell, and W. S. Hopkins, J. Am. Soc. Mass Spectrom. 
31, 405 (2020).

${ }^{45}$ W. S. Hopkins, Mol. Phys. 113, 3151 (2015).

${ }^{46}$ J. L. Campbell, A. M.-C. Yang, L. R. Melo, and W. S. Hopkins, J. Am. Soc. Mass Spectrom. 27, 1277 (2016).

${ }^{47}$ S. W. C. Walker, A. Mark, B. Verbuyst, B. Bogdanov, J. L. Campbell, and W. S. Hopkins, J. Phys. Chem. A 122, 3858 (2017).

${ }^{48}$ F. A. Londry and J. W. Hager, J. Am. Soc. Mass Spectrom. 14 (2003).

${ }^{49}$ K. St $\varnothing$ chkel, B. F. Milne, and S. B. Nielsen, J. Phys. Chem. A 115, 2155 (2011).

${ }^{50}$ J. A. Wyer and S. Brøndsted Nielsen, Angew. Chem. Int. Ed. 51, $10256(2012)$

${ }^{51}$ M. J. Frisch, G. W. Trucks, H. B. Schlegel, G. E. Scuseria, M. A. Robb, J. R. Cheeseman, G. Scalmani, V. Barone, B. Mennucci, G. A. Petersson, H. Nakatsuji, M. Caricato, X. Li, H. P. Hratchian, A. F. Izmaylov, J. Bloino, G. Zheng, J. L. Sonnenberg, M. Hada, M. Ehara, K. Toyota, R. Fukuda, J. Hasegawa, M. Ishida, T. Nakajima, Y. Honda, O. Kitao, H. Nakai, T. Vreven, J. A. Montgomery, Jr., J. E. Peralta, F. Ogliaro, M. Bearpark, J. J. Heyd, E. Brothers, K. N. Kudin, V. N. Staroverov, R. Kobayashi, J. Normand, K. Raghavachari, A. Rendell, J. C. Burant, S. S. Iyengar, J. Tomasi, M. Cossi, N. Rega, J. M. Millam, M. Klene, J. E. Knox, J. B. Cross, V. Bakken, C. Adamo, J. Jaramillo, R. Gomperts, R. E. Stratmann, O. Yazyev, A. J. Austin, R. Cammi, C. Pomelli, J. W.
Ochterski, R. L. Martin, K. Morokuma, V. G. Zakrzewski, G. A. Voth, P. Salvador, J. J. Dannenberg, S. Dapprich, A. D. Daniels, Ö. Farkas, J. B. Foresman, J. V. Ortiz, J. Cioslowski, and D. J. Fox, Gaussian 16 Revision B.01, gaussian Inc. Wallingford CT 2016

${ }^{52}$ F. Neese, WIRES Comp. Mol. Sci. 2, 73 (2012).

${ }^{53}$ M. Kállay, Z. Rolik, J. Csontos, P. Nagy, G. Samu, D. Mester, I. Ladjánszki, L. Szegedy, B. Ladóczki, K. Petrov, M. Farkas, and B. Hégely, MRCC, A Quantum Chemical Program Suite, www.mrcc.hu.

${ }^{54}$ M. Head-Gordon and T. Head-Gordon, Chem. Phys. Lett. 220, 122 (1994).

${ }^{55}$ J.-D. Chai and M. Head-Gordon, Phys. Chem. Chem. Phys. 10 6615 (2008).

${ }^{56}$ J. T. H. Dunning, Jr., J. Chem. Phys. 90, 1007 (1989).

${ }^{57}$ C. Hättig and F. Weigend, J. Chem. Phys. 113, 5154 (2000).

${ }^{58}$ A. K. Dutta, M. Saitow, B. Demoulin, F. Neese, and R. Izsák, J. Chem. Phys. 150, 164123 (2019).

${ }^{59}$ F. Santoro, A. Lami, R. Improta, J. Bloino, and V. Barone, J. Chem. Phys. 128, 224311 (2008).

${ }^{60}$ A. V. Marenich, C. J. Cramer, and D. G. Truhlar, J. Phys. Chem. B 113, 6378 (2009).

${ }^{61}$ T. Yanai, D. P. Tew, and N. C. Handy, Chem. Phys. Lett. 393, 51 (2004).

62 J. N. Bull, C. W. West, C. S. Anstöter, G. da Silva, E. J. Bieske, and J. R. R. Verlet, Phys. Chem. Chem. Phys. 21, 10567 (2019). 


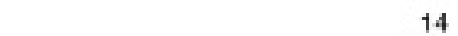

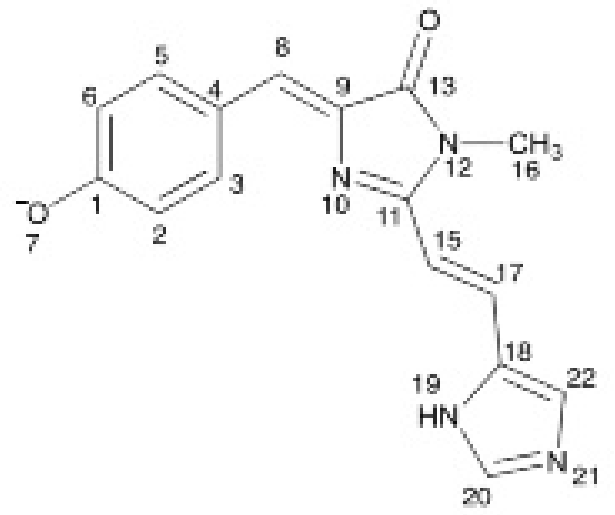

01(-)

$E_{\text {rel }}=2.3 \mathrm{kcal} \mathrm{mol}^{-1}$

(9.6 kJ mol'1)

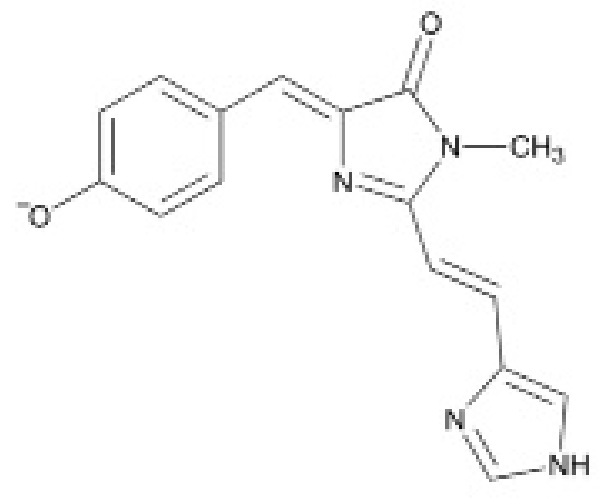

O2(-)

$E_{\text {rel }}=5.3 \mathrm{kcal} \mathrm{mol}^{-1}$

$\left(22.2 \mathrm{~kJ} \mathrm{~mol}^{-1}\right)$

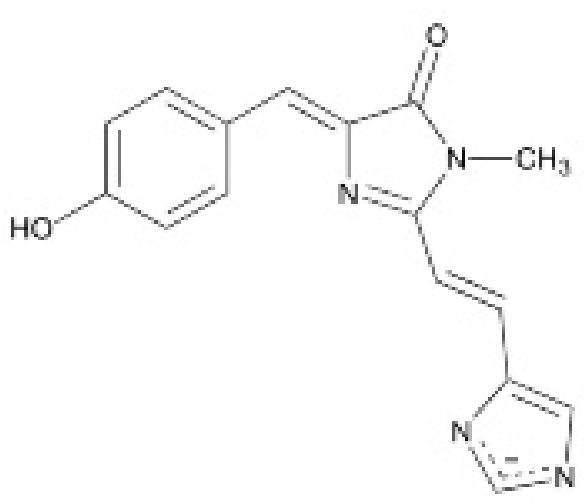

$\mathrm{N}(-)$

$E_{\text {rel }}=0.0 \mathrm{kcal} \mathrm{mol}^{-1}$ 

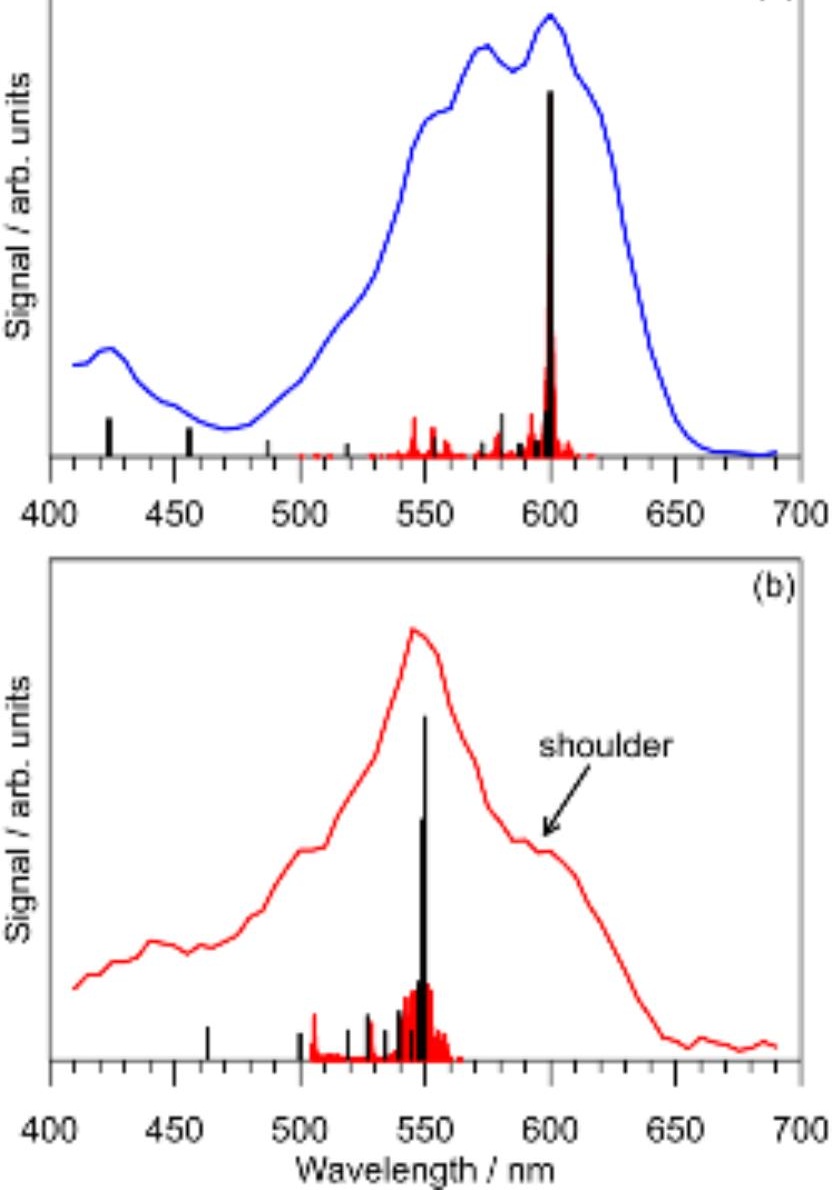

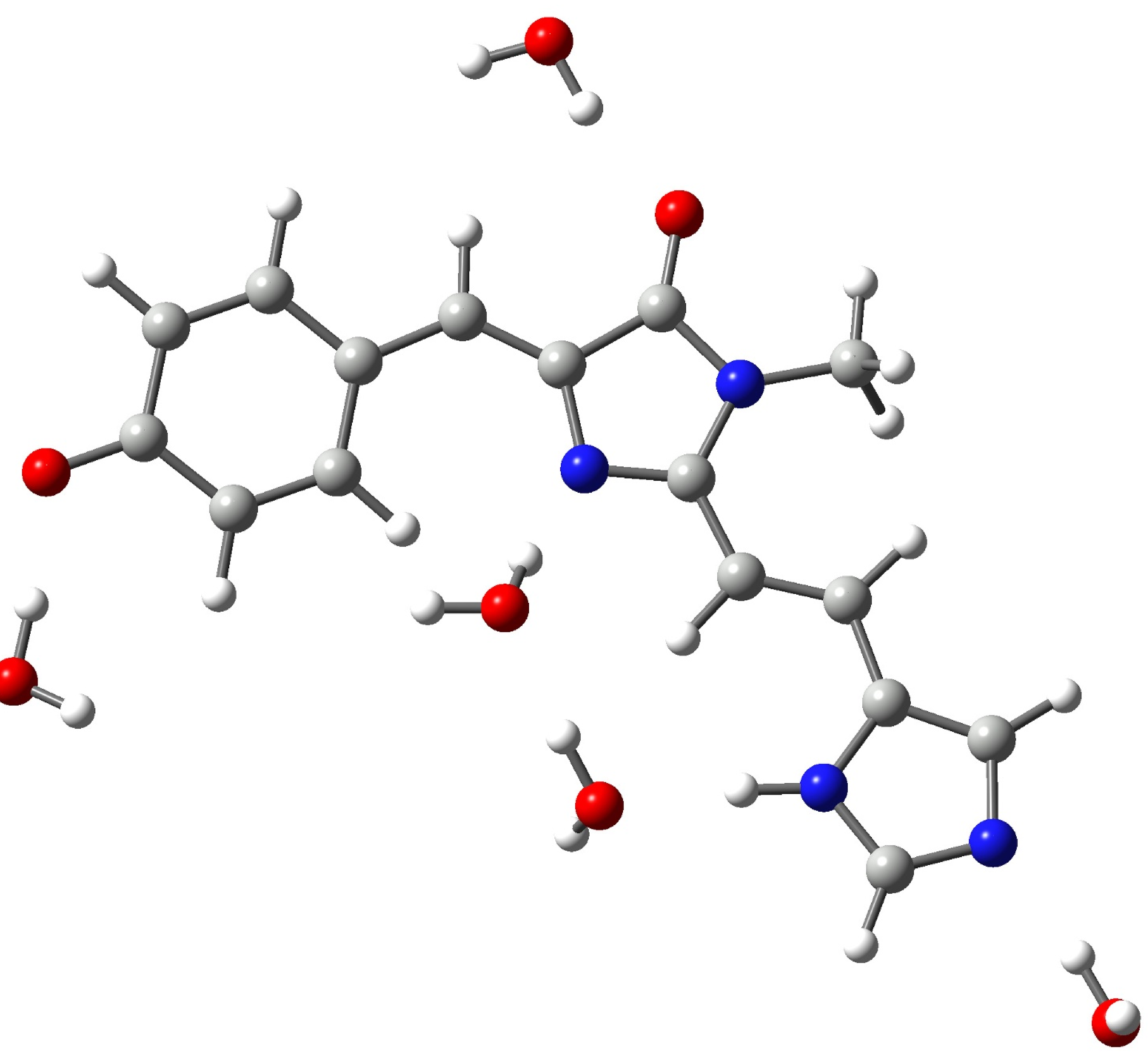